\title{
Lidil
}

Revue de linguistique et de didactique des langues

\section{Gestion de la polyphonie et figure de l'auteur dans les parties théoriques de Rapports de stage}

Fanny Rinck

\section{OpenEdition}

\section{Journals}

Édition électronique

URL : http://journals.openedition.org/lidil/23

DOI : $10.4000 /$ lidil.23

ISSN : $1960-6052$

\section{Éditeur}

UGA Éditions/Université Grenoble Alpes

\section{Édition imprimée}

Date de publication : 1 décembre 2006

ISBN : 2-914176-15-5

ISSN : $1146-6480$

\section{Référence électronique}

Fanny Rinck, «Gestion de la polyphonie et figure de l'auteur dans les parties théoriques de Rapports de stage », Lidil [En ligne], 34 | 2006, mis en ligne le 07 avril 2008, consulté le 19 avril 2019. URL http://journals.openedition.org/lidil/23 ; DOI : 10.4000/lidil.23

Ce document a été généré automatiquement le 19 avril 2019

(C) Lidil 


\title{
Gestion de la polyphonie et figure de l'auteur dans les parties théoriques de Rapports de stage
}

\author{
Fanny Rinck
}

1 Notre étude s'intéresse à la gestion de la polyphonie dans les rapports de stage d'étudiants du second cycle: centrée sur les parties théoriques de ces rapports, elle propose de décrire la manière dont les étudiants se réfèrent à d'autres travaux, et ce que manifestent ces modes de référencement en termes de figure de l'auteur dans leur texte.

2 Le public concerné suit une option professionnalisante de didactique du français langue maternelle, dans laquelle il est demandé de réaliser un stage d'observation qui se finalise par l'écriture d'un rapport. Les objectifs assignés à ce dernier sont de mener à bien une démarche réflexive: il s'agit pour les étudiants de questionner les pratiques d'enseignement et d'apprentissage du français observées en classe à l'aune des savoirs élaborés dans le champ des recherches en didactique et dans des disciplines dont la didactique réinvestit les apports. La partie théorique, réalisée en amont du stage, doit rendre compte de l'appropriation de discours "déjà-là ", où sont préconstruits des concepts et des interprétations du problème qu'a choisi de traiter l'étudiant; cette appropriation doit favoriser la construction d'un point de vue problématisé, i.e. qui permette d'analyser le problème en des termes proprement didactiques'.

3 Nous commencerons par envisager les caractéristiques attendues de la partie théorique du rapport de stage en termes de polyphonie et de figure de l'auteur, pour analyser alors les pratiques scripturales des étudiants.

4 Les parties théoriques de neuf rapports de stage ${ }^{2}$ ont été collectées dans leur version finale, après l'évaluation faite par les enseignants. Elles sont décrites au moyen d'une grille d'analyse des modes de référencement aux sources. Celle-ci est établie à partir de différents travaux menés sur la question et met l'accent sur les aspects suivants :

- les bibliographies et la systématisation ou non du renvoi aux sources dans le texte ;

- les formes et fonctions qu'y prennent les emprunts ; 
- et enfin leur insertion textuelle et la construction d'un point de vue d'auteur.

5 l'évaluation de ces parties théoriques, l'objectif étant de mieux cerner les difficultés des étudiants au regard des exigences du genre, pour déterminer en dernière instance comment les aider à produire des textes conformes aux attentes.

Polyphonie et figure de l'auteur dans le Rapport de stage

L'hétérogénéité des discours et des points de vue - qu'on traitera linguistiquement sous l'angle de la notion de polyphonie - est une dimension essentielle de la partie théorique du rapport : l'étudiant doit se référer à des sources diverses (travaux de didactique, mais aussi discours d'enseignants, de manuels, instructions officielles), les confronter et les intégrer à son questionnement. Plus précisément, il convient de dire qu'il construit son questionnement à partir de ces sources plutôt qu'il n'intègre ces sources à son questionnement : l'intégration des sources n'est que la résultante attendue, dans le texte final, du travail d'appropriation. Elle implique de la part de l'étudiant de se présenter dans son texte comme une instance responsable du sens et de construire énonciativement un point de vue dans la multiplicité des voix référencées ${ }^{3}$.

7 Le grand nombre de travaux menés, dans des genres universitaires divers, sur les pratiques scripturales étudiantes convergent vers un intérêt fort pour ces questions de polyphonie, de gestion des références, d'« images du scripteur » (Delcambre et Reuter, $2002^{4}$ ) et montrent l'importance de les travailler avec les étudiants. En défendant l'idée, à l'instar de nombre de ces travaux, que ces aspects énonciatifs doivent être envisagés en lien avec les savoirs, nous introduisons la notion de figure de l'auteur pour désigner l'instance de prise en charge modale des contenus assertés dans le texte, telle qu'elle se constitue dans la multiplicité des voix'. Il s'agit d'une caractéristique textuelle spécifique à chaque genre : figure d'un extérieur au texte qui s'y présente comme son responsable, elle est le corrélat textuel du rôle investi par le scripteur dans un genre donné et participe donc des contraintes à respecter pour produire un texte conforme aux attentes.

8 Les attentes relatives au genre du rapport de stage, et plus précisément à la partie théorique de ce rapport, si l'on s'en tient, du moins dans le contexte donné, au discours des enseignants, sont fondées sur deux exigences fortes qui reproduisent pour partie les caractéristiques des écrits de recherche: problématiser et produire un texte multiréférencé. Les descriptions faites des pratiques expertes de l'écrit de recherche doivent ainsi permettre de mieux spécifier ces caractéristiques, au niveau des formes et des fonctions des références (Boch et Grossmann, 2002 ; Grossmann, 2002), des traits de la figure de l'auteur liés à la gestion scientifique des

références ${ }^{6}$, et de la constitution d'une posture de "surénonciation"» (Rabatel, 2004), la hiérarchisation des points de vue se présentant de telle sorte que le point de vue du scripteur domine le jeu énonciatif.

9 Le rapport de stage relève de ce que Yves Reuter définit comme « l'écrit de recherche en formation " (2001, 2004). En tant que genre didactique, il doit a priori être différent de l'écrit de recherche professionnel, mais la posture réflexive qu'il requiert pour être évalué comme réussi suppose une acculturation à des normes propres aux écrits de recherche. Aider les étudiants à entrer dans ce type d'écrits suppose comme préalable de décrire leurs pratiques: il s'agit d'abord de mieux cerner les obstacles éventuels qu'ils rencontrent, à partir des dysfonctionnements ${ }^{8}$ observables dans leurs textes. Ces dysfonctionnements sont envisagés comme tels au regard des attentes du genre du 
rapport de stage, c'est-à-dire par rapport à des normes textuelles largement fondées, par hypothèse, sur celles de l'écrit de recherche, et à la fonctionnalité de ces normes dans la production et la diffusion du savoir. Ces descriptions doivent alors permettre de s'interroger sur les attentes et de déterminer dans une perspective interventionniste quel modèle de référence convoquer en termes de gestion de la polyphonie et de figure de l'auteur.

Analyse des pratiques scripturales des étudiantsVariabilité dans les modes de renvoi aux sources

10 Le premier dysfonctionnement observable dans les parties théoriques des rapports de stage concerne la référenciation des sources. Si l'on met de côté pour l'instant le problème de défaut de notation des sources, pour s'en tenir aux références effectives dans le corps du texte et dans la bibliographie, on remarque une variabilité interindividuelle des stratégies de notation des sources ainsi qu'une variabilité à l'intérieur d'une même production. Les variations concernent les éléments mentionnés (nom d'auteur, titre de l'ouvrage, année, page, etc.) et leur insertion typographique. Elles posent un problème d'exhaustivité des références et d'absence de systématicité. Cette variabilité des modes de référencement ne peut être rapportée aux distinctions en usage quant à la nature de la source consultée (article, revue ou ouvrage), ni quant au type de reprise des textes lus (citation et reformulation) et à l'insertion syntaxique de la référence dans le corps du texte (référence apposée entre parenthèses ou intégrée à la syntaxe); au contraire même, elle tend parfois à rendre confuses ces distinctions.

11 Concernant les bibliographies, le lieu d'édition n'est pas systématiquement mentionné, de même que les pages des articles; pour trois d'entre elles, le caractère aléatoire des modes de notation nuit à la distinction entre article et ouvrage, sans compter que l'absence de systématicité affecte également le rapport entre texte et bibliographie, toutes les références convoquées dans le texte n'étant pas reprises dans la bibliographie et inversement.

12 Dans le corps du texte, les pages des sources consultées ne sont presque jamais mentionnées. La date n'y est précisée systématiquement que dans le texte 3 et se trouve la plupart du temps omise; elle est parfois remplacée par le titre de l'ouvrage, comme dans le texte 1 où l'on note une triple alternative : précision de la date, du titre, ou des deux. La seule insertion des noms d'auteurs fait état de cette variabilité : dans toutes les parties théoriques interviennent de manière manifestement aléatoire le nom et le prénom, le nom et l'initiale, ou encore le nom seul et ce, que la référence soit ou non placée entre parenthèses; dans le texte 5 , ces noms sont tantôt soulignés tantôt non. Dans le texte 9, ce sont les titres qui font l'objet de procédés typographiques alternatifs : placés entre guillemets, ils se présentent parfois aussi en italique.

13 S'il n'est pas possible d'aborder la question du renvoi aux sources autrement que sous un angle normatif, en termes de manques ou d'écarts par rapport à un modèle de référence, il n'en convient pas moins de souligner que la notation des sources, telle que la pratiquent les étudiants, tend parfois à nuire à sa fonctionnalité. Au-delà des difficultés posées, et pas seulement aux étudiants, par la diversité des normes en matière de référencement bibliographique, ce problème de fonctionnalité signale l'adoption partielle d'un modèle de référence explicité par les enseignants, et qui ne devrait pas a priori faire obstacle en termes de compétences linguistiques.

Défaut de notation des sources 

de réception: la disparition des sources, légitimement taxée de plagiat par les enseignants qui reconnaissent dans les productions de leurs étudiants des extraits voire des pans entiers de propos qui sont empruntés à d'autres sans être signalés comme tels. Six des parties théoriques font ainsi l'objet de corrections relatives à ce défaut de notation des sources, notamment le texte 9 : «Jolibert, citez vos sources ", «quelles sont vos références?? », ou encore le 5 : «problèmes réels de référenciation du discours d'autrui », "gros problèmes d'identification des sources », « il faut davantage citer les sources », " vous frisez le plagiat ». marquer comme tel, la part masquée de l'hétérogénéité discursive est pour ainsi dire inévitable : elle reflète le travail d'appropriation des discours lus qui, digérés dans la toile d'araignée des reformulations successives (Brey, cité par Frier, 1998 : 67) peuvent ne plus être donnés à voir comme autres. Il ne s'agit pas pour autant de nier que le défaut de notation des sources peut résulter d'un acte conscient, et qu'il est des cas où plutôt qu'un quelconque travail d'appropriation, c'est le copier/coller qui résume le passage des textes lus à un nouveau texte. La disparition des sources semble cependant pouvoir être comprise en partie au moins comme une difficulté du scripteur à parler en son nom propre alors qu'il bâtit l'ensemble de son écrit avec des textes lus.

16 Ainsi, le caractère technique du sujet abordé dans le texte 5 (les aspects psycho-moteurs du développement du geste graphique) et le fait que le scripteur du texte 9 travaille sur la "démarche de chantier» pour former les apprenants à l'écriture en s'appuyant largement sur l'ouvrage de Josiane Jolibert qui formalise cette démarche, peuvent expliquer l'obstacle que représente alors la nécessité de rendre compte de chaque emprunt. On retrouve, à travers le défaut de notation des sources, une conception que met en évidence Isabelle Laborde-Milaa (2002: 191) selon laquelle parler en son nom propre équivaut pour les étudiants à ne plus citer ni reformuler. Les difficultés que suscite le fait de devoir écrire en son nom propre mais à partir des textes lus, traduisent ainsi une interprétation problématique des attentes de l'évaluateur pour qui ces deux exigences ont complémentairement du sens, en termes de texte à produire et de construction des savoirs.

17 Linguistiquement, les propos des auteurs lus sont ainsi repris sur le mode de l'appropriation maximale (Delamottte Legrand, 1996), i.e. sans que rien, d'un point de vue énonciatif, ne signale qu'il y a deux voix - ce qui obère le statut d'énonciation située de ces propos, pour n'en retenir que la valeur informative. On observe le même effet dans le maniement de données chiffrées dont la source énonciative semble relever de la « rumeur des discours» (Fabre-Cols, 2001), comme dans le texte $2:$ «il apparait de surcroit que cinquante pour cent des Français ne lisent rien ». En l'absence de mention de la source, les données chiffrées se voient attribuer en elles-mêmes une autorité et participent en ce sens d'une rhétorique de la scientificité; se pose ainsi le problème de l'image que donne le texte de son auteur en termes de rapport au savoir, même si ne pas citer ses sources ne saurait préjuger à soi seul des attributs de ce rapport. Contraire aux attentes du genre, le fait de passer sous silence le statut énonciatif des assertions empruntées à d'autres témoigne en tout état de cause d'une gestion non scientifique des références et montre que le renvoi aux sources est une question d'acculturation aux normes propres aux genres universitaires, acculturation qui, même si l'on s'en tient aux bibliographies, est loin d'aller de soi. 
Formes et fonctions des discours autres

18 L'analyse des formes et des fonctions des références à d'autres discours dans les parties théoriques rejoint les constats établis dans les travaux menés sur les pratiques d'écriture des étudiants, quel que soit le genre concerné. Concernant les modes de représentation des discours autres, l'emprunt explicite peut prendre la forme de citations ou de reformulations. Françoise Boch et Francis Grossmann (2002) l'ont bien montré, la stratégie d'évocation ou d'allusion, consistant à limiter la référence à un nom d'auteur, à un ouvrage ou à un courant, sans rendre compte du discours lui-même, est une stratégie très rare dans les pratiques étudiantes. De telles désignations en vigueur dans les pratiques expertes, et d'ailleurs plus représentatives de la gestion des références dans leurs textes que le discours rapporté (Grossmann, 2002), requièrent une bonne connaissance du champ; elles manifestent en outre une posture auctoriale de détermination des paradigmes (Grossmann et Rinck, 2004) qui pourrait, comme le précisent Françoise Boch et Francis Grossmann (2002), être perçue comme dommageable de la part de scripteurs néophytes dont on attend davantage d'explicitations quant aux contenus des textes de référence.

Du côté des fonctions privilégiées, si l'on se réfère à la typologie proposée par ces deux auteurs et à celle, très proche, de Mohamed Kara (2004), on remarquera que les emprunts explicites dans les parties théoriques ont pour vocation première d'introduire une idée et, inversement, d'appuyer une affirmation (vingt-sept références sur quarante-huit remplissent l'une ou l'autre de ces fonctions). Dans le même sens, l'emprunt à visée définitoire est également très représenté (à hauteur d'un tiers des références explicites). Si cette distribution relativement conforme à ce qui a déjà été montré dans les travaux existants fait apparaitre que les citations dialectiques ou doctrinales vouées à l'instruction d'un débat sont absentes, notons qu'il s'agit là encore de traits caractéristiques d'une posture «surénonciative» d'expert (Grossmann et Rinck, 2004), qu'il serait en cela sans doute peu pertinent de vouloir ériger en modèle de référence des pratiques scripturales étudiantes.

20 Par rapport aux dimensions qu'il semble souhaitable de travailler avec les étudiants dans une perspective de didactisation des genres universitaires, il est intéressant de constater que les textes analysés font preuve d'une diversification des stratégies, tant au niveau des modes de représentation des discours autres que de leur fonction. Dans toutes les parties théoriques, on observe que citations et reformulations alternent. Les citations ellesmêmes prennent la forme d'ilots et de pavés citationnels. Quant aux modes d'introduction des emprunts, quoiqu'ils indiquent une préférence pour des insertions relativement figées du type "selon $\mathrm{X}$ ", ils reflètent également une tendance des étudiants à exploiter une palette de possibilités variées, où le discours direct, perçu comme maladroit, reste très marginal. Au vu des résultats des travaux existants, cette tendance à la diversification des modes et fonctions de la représentation de discours autres, méritoire, pourrait sans doute s'expliquer comme le fruit de l'accompagnement méthodologique dont ont bénéficié les étudiants, en particulier sur cette question importante, selon les enseignants, de l'intégration des discours autres.

21 C'est manifestement au niveau de la place faite aux discours autres que les parties théoriques analysées se distinguent le plus: les références explicites aux textes lus montrent deux tendances diamétralement opposées, déjà mises en évidence par Yves Reuter (2001:17) : l'accumulation et la restriction. Ainsi, la partie théorique 5 posant, on l'a dit supra, un problème de plagiat, se caractérise par une restriction des emprunts 
signalés comme tels, qui se limitent à sept. Conséquence directe du défaut de notation des sources dans ce texte, cette tendance se retrouve cependant dans les parties théoriques 2, 3 et 9 (respectivement huit, neuf et dix références explicites dans le corps du texte), où elle ne semble pas tenir non plus au nombre de sources consultées.

Ce faible nombre d'emprunts signalés, au regard des autres parties théoriques, pourrait alors s'expliquer en 2 et 9 par la présence de reformulations que l'on peut dire globales, lorsque les étudiants disent au début d'une partie s'appuyer sur un ouvrage de référence, sans repréciser ensuite systématiquement que chaque assertion est imputable à cette autre voix. On aurait dans ces cas une forme de co-énonciation, au sens où l'entend Alain Rabatel (2004), de prise en charge double des contenus assertés, l'énonciateur se faisant dans l'ensemble d'une partie le relais de propos empruntés avec lesquels il manifeste ainsi son accord tacite. Une telle posture énonciative est a priori inédite chez les experts, au moins sous cette forme : elle peut intervenir mais de manière très localisée, un certain nombre de procédés permettant au scripteur de faire en sorte que sa voix l'emporte ${ }^{10}$ ou reprenne rapidement le dessus.

La tendance à l'accumulation des emprunts trouve quant à elle une illustration frappante dans la partie théorique 7. A l'exception de l'introduction et de la conclusion, l'ensemble du texte 7 se construit à partir de citations extraites des textes lus: on y compte ainsi soixante-douze références à d'autres textes. Là encore le nombre de sources documentaires n'est pas en cause, puisqu'elles se limitent à quatre, de sorte que le taux d'exploitation d'une même source est extrêmement élevé. Si la tendance à l'accumulation, telle qu'elle a été mise en valeur dans un ensemble de travaux", prend souvent la forme de bribes juxtaposées, l'«ostension» (Reuter, $2001: 18)$ des discours autres dans la partie théorique 7 n'induit pas un effet de plaquage et ne nuit pas à leur fonctionnalisation, leur texte se présentant sur le modèle d'une synthèse menée entre les différents emprunts :

Il faut aussi s'assurer selon De la Monneraye du «volontariat réel de l'enfant à propos du soutien » et donc de ne pas lui «imposer à nouveau une démarche de l'extérieur » mais de proposer à chaque élève une méthode adaptée à ses difficultés et à sa personnalité. Boimare ajoute qu'il faudrait pour ces jeunes réenvisager les différentes formes de soutien en "[ouvrant] vers de nouveaux processus cognitifs [...] dans un cadre renforcé avec des règles impératives, pour que ces enfants se sentent rassurés et retrouvent un intérêt pour la scolarité ». Maryse Metra, rééductrice Fnaren, insiste également [...] (7).

Les scripteurs dressent ainsi un état des lieux de la question traitée en reformulant et citant les textes lus, qu'ils font dialoguer entre eux. En ce sens, l'accumulation de citations n'est pas ici préjudiciable à la construction d'une posture auctoriale, mais le fait que près de $45 \%$ des mots du texte soient des citations confère à la figure de l'auteur des attributs spécifiques: ces ilots citationnels reflètent sans doute un «fantasme de la scientificité » (Pollet et Piette, 2002: 171) et traduisent une crainte des étudiants de s'affirmer face à des auteurs qui, dans le texte produit, disent tout du sujet traité. Cette crainte est rendue tangible dans un emploi obsessionnel des guillemets, utilisés pour délimiter des mots isolés: "ce "pédagogue" ", "les "difficultés" ». Si les étudiants estiment par là signaler un emprunt, la récurrence de ce procédé distanciateur dans l'usage dénominatif rend tangible la difficulté des étudiantes à endosser l'emploi de mots communs trouvés chez d'autres ; les scripteurs évitent de parler en leur nom propre, s'en remettant à l'autorité du propos des auteurs lus. 

cependant intéressante : elle permet à l'étudiant de s'approprier les textes lus et de manipuler les concepts avant de prendre la parole avec ses mots propres, en travaillant à la construction d'une "structure hébergeante» (Kara, 2004: 119) qui intègre véritablement les emprunts. Dans une perspective d'intervention didactique, exploiter une telle étape pourrait permettre de mieux sérier les exigences et de les hiérarchiser, pour pallier les effets d'inhibition ressentis par certains étudiants face à des contraintes multiples, parfois perçues comme confuses ou contradictoires ${ }^{12}$; que l'on travaille avec les mots de l'autre ou qu'on les reformule, c'est cette question de l'insertion textuelle des emprunts qui est, nous semble-t-il, la plus déterminante dans la construction d'un point de vue d'auteur et dans le travail sur les concepts.

La construction polyphonique d'un point de vue d'auteur autres, notamment ceux entrepris par Françoise Boch et Francis Grossmann (2001b), Alain Rabatel (2002), et Mohamed Kara (2004), ont mis en évidence les difficultés qu'ils ont dans la gestion de la cotextualisation des emprunts: les pratiques d'écriture des étudiants font état de ce point de vue de problèmes d'enchainement, voire de décalages argumentatifs, face auxquels Mohamed Kara propose un certain nombre d'outils à même de favoriser l'activité d'expansion attendue à partir d'une citation pour en exploiter le caractère heuristique dans son texte. Les parties théoriques ici analysées n'échappent pas aux dysfonctionnements observés quant à la cohérence textuelle.

Sans aborder dans le détail ces dysfonctionnements déjà bien décrits, liés à l'insertion textuelle des emprunts, nous en évoquerons cependant certains aspects en nous centrant sur le problème de la construction du point de vue dans la multiplicité des instances énonciatives : comment le scripteur se positionne-t-il, d'un point de vue énonciatif et épistémique, dans un texte multi-référencé ?

Se positionner suppose d'abord une distinction nette des voix en présence, nécessaire à la cohérence polyphonique (Fløttum, 2002) du texte. Or si le plagiat ignore cette distinction en présentant comme le fait d'une seule voix une assertion empruntée à une source masquée, les travaux évoqués l'ont souligné, les emprunts signalés comme tels dans les écrits d'étudiants se caractérisent parfois par des brouillages énonciatifs. C'est le cas dans cet extrait tiré de la partie théorique 9 :

les chantiers sont "centrés sur un type de texte » poursuit J. Jolibert. Rappelons que nous entendons par "type de texte» les types d'écrits sociaux les plus courants. Un chantier peut être le projet de faire «une lettre de demande, un compte-rendu, une invitation... » (9).

La confusion des voix ressortit dans cette séquence au caractère indécis des marques typographiques des guillemets et de l'italique, ainsi qu'à un "nous ", qui instaure une prise en charge énonciative où se confondent la voix de l'étudiant, la voix de l'étudiant s'accordant avec celle de l'auteur, et la voix de l'auteur, reprise à son compte et non signalée comme telle par l'étudiant. Ce passage porte la trace du travail d'appropriation et du passage des textes lus à une prise de parole personnelle, mais demanderait à être retravaillé, pour que la co-énonciation soit formellement marquée comme telle, en tant que prise en charge double fondée sur une démarcation des deux voix en présence. L'absence de démarcation fait obstacle à la construction d'une posture énonciative autonome et rejoint le constat mis en évidence par Marie-Christine Pollet et Valérie 
Piette (2002 : 177) des difficultés des étudiants à instaurer une relation dialogique et à se présenter comme constructeurs de sens dans leur texte.

La seule identification des voix garantit leur distinction et la construction par là d'une posture énonciative autonome que l'on trouve dans des formes d'emprunts tout à fait canoniques du type « comme dit X » ou «selon X ». Mais demander aux étudiants de « se positionner » dans leur texte ne va pas sans présupposés quant à leurs connaissances de ces aspects énonciatifs. Or cette exigence se trouve interprétée de manière restrictive en termes de prise de position explicite : certains étudiants en retiennent qu'il s'agit de prendre parti. Cette idée que se positionner requiert de signaler explicitement son accord ou son désaccord peut donner lieu à des stratégies dommageables, comme on le voit dans la partie théorique 7. On y observe en effet une forme d'accord et une forme de désaccord toutes deux problématiques.

L'expression de l'accord dans «nous soutenons totalement la pensée de Boimare et de Métra » manifeste une maladresse d'ordre phraséologique qui n'est pas sans conséquence sur la figure de l'auteur en raison du degré d'adhésion qu'elle induit. L'enseignant conseille une autre formulation "nous adhérons sans réserve à l'analyse de », proposant ainsi de substituer à l'adhésion « totale » - sur le mode du « soutien » - à la « pensée » des auteurs, une adhésion épistémique à ce qui relève de l'ordre du construit ("l'analyse »).

La forme de désaccord intervenant dans cette partie théorique passe également par une prise en charge personnelle :

nous préférons la vision de Boimare et Métra [à celle de Perron, Aublé, Compas] car nous pensons que considérer un enfant dans son statut d'élève l'aide à devenir ou à se sentir «élève" alors que le terme de «sujet» rappelle celui de «sujet de laboratoire » qui ne fait que subir la situation qu'il endure et qui ne peut être autonome (7).

Le problème ici tient à ce que ce désaccord repose sur une distorsion du propos des auteurs lus, propos cité plus haut dans la partie théorique : «[Péron, Aublé et Compas] appuient que "l'enfant est une personne, c'est seulement dans une telle perspective que l'on peut lui donner pleinement son statut de sujet" ». Le positionnement s'avère ainsi très artificiel : il repose sur une opposition terminologique de « statut d'élève » et « statut de sujet » qui rend possible l'opposition entre les positions des auteurs; les étudiants se détachent donc sémantiquement du propos cité pour servir une visée d'ostension d'un désaccord.

S'il s'agit pour les étudiants d'adapter le texte à produire par rapport à l'image qu'ils s'en font, cette idée qu'il faut prendre parti entre en conflit avec l'image que les scripteurs ont d'eux-mêmes dans la tâche, en raison des difficultés qu'ils éprouvent à se sentir autorisés à discuter les propos des auteurs lus. La partie théorique 7, qui se caractérise doublement par cette prise de position artificielle et par une profusion citationnelle, porte ainsi les traces de ces images du genre et de soi qui se bousculent dans la production du texte, et de la tension que doivent gérer les scripteurs entre montrer l'autre et se montrer en tant qu'auteur.

Conclusion

35 L'écriture de la partie théorique pose la question de l'acculturation à un genre dont les caractéristiques énonciatives se veulent en grande partie codifiées en regard des normes propres à l'écrit de recherche. Cette acculturation soulève différents problèmes qui tiennent principalement aux difficultés qu'ont les étudiants à se représenter dans une position d'auteur, d'une part, et à se présenter comme auteurs dans leur texte, d'autre 
part. Au seul niveau de la bibliographie, le modèle de référence, a priori accessible en termes de compétences scripturales, n'en est manifestement pas un pour les étudiants.

Aussi, l'enseignement de ces normes ne saurait se limiter à une approche techniciste, c'est même là le fondement consensuel donné au projet d'une didactique de l'écrit dans l'enseignement supérieur (Delcambre et Jovenet, 2002). Il requiert par ailleurs de mieux déterminer quel modèle de référence convoquer en termes de figure de l'auteur dans le genre du rapport de stage. En raison de leur statut objectif et de l'image qu'ils se font d'eux-mêmes comme scripteurs, il est peu pertinent, en effet, d'attendre des étudiants de licence qu'ils adoptent une posture surénonciative caractéristique des pratiques expertes. Celle-ci suppose une connaissance avancée du champ, une intériorisation de routines scripturales propres aux écrits de recherche, mais aussi une «illusio » (Bourdieu, 1997 : 122) spécifique par laquelle le scripteur joue le jeu du genre, se présentant dans son texte comme un expert, garant de ce qui est dit, et qui n'a de cesse d'affirmer sa singularité de chercheur.

Prioritairement, il importe donc d'expliciter et de hiérarchiser des attentes qui, pour avoir complémentairement $\mathrm{du}$ sens pour le chercheur, restent obscures voire contradictoires pour le néophyte. Des exigences comme se positionner et marquer sa distance restent telles quelles pour le moins problématiques, car elles se fondent conjointement sur des critères textuels (leur assise énonciative) et extra-textuels (le rapport au savoir). La description même des pratiques des étudiants, en tant que préalable nécessaire à l'accompagnement didactique, demanderait à éviter de mettre sur le même plan les divers dysfonctionnements observables dans les textes. Par exemple, la profusion de citations, extrêmement dommageable quand elle donne lieu à des textes décousus, pourrait aussi représenter une étape qui permet de travailler, à partir d'ilots citationnels, à la construction d'un texte cohérent.

Quelles stratégies peut-on donc raisonnablement exiger de la part des étudiants pour qu'ils se positionnent en tant qu'auteurs dans leur texte, dans la multiplicité des voix convoquées? Parmi d'autres pistes, c'est d'abord l'identification des voix en présence qui nous semble devoir être travaillée : il s'agirait de dépasser l'interprétation du positionnement en termes de prise de position en rendant les étudiants davantage conscients de l'assise énonciative de cette exigence. L'identification des voix permet en effet que se construise une posture énonciative autonome, distincte de celle des auteurs lus, quitte à ce que les contenus soient assertés, temporairement au moins, sur le mode de la co-énonciation (Rabatel, 2004).

D'autre part, si l'activité d'expansion à partir des emprunts mérite d'être constituée en objet d'enseignement, il nous semble qu'un tel travail gagnerait à s'appuyer sur la confrontation des points de vue des auteurs lus. Approfondir la question en la cernant de différents points de vue représente en effet une manière intéressante d'exploiter le potentiel heuristique des emprunts aux textes lus et de trouver sa propre voix ${ }^{13}$ : à partir des éléments retenus des textes lus, le fait de faire dialoguer les auteurs entre eux produit un point de vue énonciatif et épistémique conforme aux caractéristiques attendues de nuance, de distance et de complexité. Un travail sur ces formes de confrontation des points de vue permettrait d'éviter les problèmes posés par des exigences aporétiques pour l'étudiant et de donner à l'écriture le rôle d'outil dans la construction d'une réflexion à la fois personnelle et documentée. 


\section{BIBLIOGRAPHIE}

Delamotte-Legrand, R. (1996) : Polyphonie dans l'écriture, Cahiers du français contemporain, 3 , 193-210.

DELCAMBRe, I. et JOVEnET, A.-M. (dir.), (2002) : Lire-écrire dans le supérieur, Spirale, 29, Lille 3.

DELCAMBRE, I. et REUTER, Y. (2002) : Images du scripteur et rapports à l'écriture, Pratiques, 113-114, 7-28.

LABORDE-MILAA, I. (2002) : Polyphonie énonciative. Représentations d'étudiants en position d'évaluateurs de leurs pairs, Spirale, 29, 181-200.

LABORDE-MILAA, I., BOCH, F. et REUTER, Y. (dir.), (2004) : Les écrits universitaires, Pratiques, 121-122, Metz, Cresef.

\section{NOTES}

1. Notons que l'élaboration de cet écrit fait l'objet d'un accompagnement méthodologique, fondé sur l'observation de rapports antérieurs, sur des apports concernant la démarche de problématisation, et sur un suivi personnalisé des étudiants par le biais d'écrits intermédiaires (fiches relatives à l'avancement de la problématique, que nous n'examinerons pas ici).

2. Les parties théoriques sont ici référencées de 1 à 9 . Certaines ont été produites en binôme, mais nous n'exploiterons pas ici la différence potentielle entre ces textes et les productions individuelles.

3. On restreint l'analyse de la polyphonie aux formes d'hétérogénéité montrée (AuthierRevuz, 1984) où un point de vue est référencé, c'est-à-dire montré comme autre en étant indexé à une source locutoire, en tenant compte cependant aussi de la question de la disparition de cette source dans le cas du plagiat.

4. Outre les travaux sur les images du scripteur menés par l'équipe Théodile à Lille3, citons notamment Régine Delamotte-Legrand (1996), Catherine Frier (1998), Françoise Boch et Francis Grossmann (2001a), Isabelle Delcambre et Anne-Marie Jovenet (2002), Marie-Christine Pollet et Françoise Boch (2002), et Isabelle Laborde-Milaa et al., (2004).

5. Attendu par le lecteur et visé par le scripteur, ce « principe d'économie dans la prolifération des voix » (Foucault, 1969) équivaut à la cohérence polyphonique du texte (Fløttum, $2002: 342$ ).

6. Par exemple dans les notes (Rabatel, $2002: 61$ ), ou au niveau des axiologiques utilisés pour évaluer un point de vue (Rinck, 2006 : 104).

7. Pour une description de cette posture comme caractéristique de l'écrit de recherche, cf. Francis Grossmann et Fanny Rinck (2004).

8. Yves Reuter (2004) propose des éléments de réflexion sur cette notion.

9. Que l'on peut nommer ainsi en référence à la notion d'hétérogénéité masquée qui s'oppose à l'hétérogénéité montrée chez Jacqueline Authier-Revuz (1984). 
10. Citons par exemple une des stratégies permettant d'éviter formellement un effet de co-énonciation : quand une référence est postposée à une assertion et introduite par une formule comme cf. aussi ou voir aussi, le scripteur se présente comme seul responsable des propos et signale cependant qu'ils ont été produits aussi par d'autres, retirant de cette stratégie le double bénéfice de la légitimité du dit et de son attribution (Grossmann et Rinck, 2004).

11. Voir notamment Yves Reuter (2001) et Marie-Christine Pollet et Valérie Piette (2002). 12. Ces aspects, bien mis en évidence par les travaux précédemment cités, sont centraux dans les entretiens menés avec les scripteurs ici concernés (Rinck, 2004).

13. Donnons-en un exemple réussi extrait de la partie théorique $1:$ : Plus concrètement, il y a diverses manières d'amener l'enfant à créer. Christiane Keller (poète) suggère d'utiliser des structures de départ pour bâtir son propre poème. Par exemple, on peut donner comme consigne de débuter son poème par "j'aime" ou "bleu comme...". Néanmoins J.-F. Massol pense qu'il ne faut pas se limiter à une utilisation mécanique des canevas pour éviter l'imitation. L'enfant est d'ailleurs capable de créer par lui-même sans se voir imposer des règles, comme le dit Gisèle Bertillot (professeur des écoles) qui propose à ses élèves d'élaborer des arbres à poésies. Cependant il ne faut pas oublier qu'un poème est constitués de rimes, de vers, de rythme... Donc, même si l'enfant est libre, il reste toujours un minimim de contraintes à respecter ».

\section{RÉSUMÉS}

Notre étude s'intéresse à la gestion de la polyphonie dans les rapports de stage d'étudiants du second cycle : elle décrit les dysfonctionnements observés dans la manière dont les étudiants se réfèrent à d'autres travaux dans la partie théorique de leur rapport, et ce que manifestent ces modes de référencement en termes de figure de l'auteur dans leur texte. Les difficultés qu'ils ont, d'une part à se représenter comme auteurs et, d'autre part, à se présenter comme auteurs dans leurs textes, suggèrent que pour aider les étudiants à entrer dans ce type d'écrits, il importe de s'interroger sur les attentes du genre et de hiérarchiser les exigences en évitant de les calquer sur les pratiques expertes. 